\title{
Um Sistema de Gerenciamento e Automação de Climatização para Eficiência Energética
}

\author{
Felipe Rocha, Luís Felipe Santos, José Gameleira Neto, Alan Fernandes, \\ Thais Batista, Everton Cavalcante \\ Universidade Federal do Rio Grande do Norte (UFRN) \\ Natal-RN, Brasil \\ \{felipebarbalho.95, santosfluis19, jgdrneto, alan.fernandes63, \\ thaisbatista, evertonranielly\}@gmail.com
}

\begin{abstract}
Resumo. O mau uso de aparelhos de ar condicionado tem sido identificado como um fator de influência significativa no desperdício de energia elétrica, devido ao seu consumo energético elevado e seu amplo uso no Brasil. Com o objetivo de otimizar o uso desses equipamentos com vistas à eficiência energética, este trabalho apresenta o Smart Place, um sistema de gerenciamento de ambientes para o controle automatizado de aparelhos de ar condicionado. Nesse sistema, sensores e câmeras de vídeo coletam dados referentes a temperatura, umidade e presença de pessoas nos espaços monitorados, de modo que tais dados são utilizados para realizar intervenções nos aparelhos de ar condicionado para evitar que eles permaneçam ligados quando o ambiente não estiver sendo realmente utilizado. Este artigo descreve o Smart Place, sua implantação e operação na Universidade Federal do Rio Grande do Norte (UFRN), em Natal-RN, e discute alguns benefícios resultantes da intervenção automática feita pelo sistema em comparação com o tradicional controle manual.
\end{abstract}

\begin{abstract}
The misuse of air conditioners has been identified as a significant influence factor on the waste of eletricity due to its high energy consumption and widespread use in Brazil. Aiming at optimizing the use of those equipments towards energy efficiency, this work presents Smart Place, an ambient management system for automated control of air conditioners. In this system, sensors and cameras collect data regarding temperature, humidity, and presence of people in the monitored spaces, so that these data are used to perform interventions in air conditioners to prevent them from remaining turned on when the place is not being used. This paper describes Smart Place, its deployment and operation at the Federal University of Rio Grande do Norte (UFRN), Natal, Brazil, and discusses some benefits resulted from the automated intervention made by the system compared to the traditional manual control.
\end{abstract}

\section{Introdução}

De acordo com a Associação Brasileira das Empresas de Serviço de Conservação de Energia (ABESCO), o Brasil desperdiçou em energia elétrica o equivalente a $\mathrm{R} \$ 61,7$ bilhões entre 2014 e 2016 [ABESCO 2017]. Esse é sem dúvida um cenário preocupante, pois tal desperdício, se evitado, poderia ter reduzido a utilização das termelétricas no decorrer do período em que as usinas hidroelétricas apresentaram baixa nos seus reservatórios, evitando assim a emissão de gases do efeito estufa e diminuindo drasticamente a taxa paga pela energia elétrica. 
Entre as possíveis causas para o péssimo aproveitamento da energia elétrica estão a desatualização do maquinário industrial, das lâmpadas e dos equipamentos eletrodomésticos. Em particular, aparelhos de ar condicionado contribuem significativamente com o alto consumo de energia elétrica, devido a sua alta utilização em decorrência das condições climáticas observadas na maior parte do ano no Brasil e a grande difusão desses equipamentos tanto em prédios residenciais quanto comerciais e acadêmicos. Essa situação agrava-se ainda mais quando tais aparelhos são utilizados de forma errônea e sem controle, como, por exemplo, nos casos em que eles permanecem ligados por várias horas em ambientes que não estão sendo utilizados.

Com vistas a propiciar a utilização de recursos de forma econômica e sustentável, diversos trabalhos buscam na automação uma forma de desenvolver sistemas de controle e monitoramento de ambientes, visando não somente a otimização do consumo energético, como também a comodidade das pessoas presentes nesses ambientes. A ideia é encontrar na eficiência energética uma forma de conter a expansão do consumo sem comprometer qualidade de vida e desenvolvimento econômico [Berriel 2017]. Nesse contexto, surge o conceito de edifícios inteligentes, que podem ser entendidos como edifícios capazes de minimizar o consumo de energia e melhorar a experiência e produtividade de usuários.

A operação de um edifício inteligente é baseada no monitoramento e controle de processos através da coleta, processamento e envio de informações utilizando sensores, atuadores e microcontroladores a partir de programações previamente definidas pelo operador. Dispondo dessas informações, um sistema de gerenciamento de ambientes poderia, por exemplo, levar em consideração fatores como temperatura, umidade e presença de indivíduos para determinar quando o aparelho de ar condicionado do ambiente deve ou não ser ligado e se deve haver elevação ou não da temperatura. Com isso, seria possível manter um controle do uso dos equipamentos e favorecer o uso eficiente da energia elétrica.

Na perspectiva de contribuir com a eficiência energética em prédios inteligentes, este trabalho apresenta o Smart Place, um sistema de gerenciamento de ambientes que permite controlar, de forma automática, aparelhos de ar condicionado, evitando assim o desperdício de energia elétrica e proporcionando maior conforto e praticidade às pessoas em tais ambientes. O Smart Place é constituído de hardware que realiza o controle dos aparelhos de ar condicionado com base em dados coletados por sensores de movimento, temperatura, umidade e câmeras de vídeo, além de contar com um módulo Web que possibilita gerenciar os dispositivos e ambientes monitorados. O sistema é uma das soluções integradas de campus inteligente desenvolvidas no âmbito do Projeto Smart Metropolis ${ }^{1}$ na Universidade Federal do Rio Grande do Norte (UFRN), em Natal-RN, estando atualmente implantado em onze salas e laboratórios da instituição.

Este artigo está estruturado da seguinte forma. A Seção 2 discorre brevemente acerca de trabalhos relacionados. A Seção 3 apresenta o Smart Place em termos de sua estrutura e fluxo de informação. A Seção 4 descreve alguns resultados obtidos da avaliação do Smart Place nos diversos locais onde está instalado, e discute sua contribuição com relação às soluções existentes. Por fim, a Seção 5 traz algumas considerações finais.

\footnotetext{
${ }^{1} \mathrm{http}: / /$ smartmetropolis.imd.ufrn.br/
} 


\section{Trabalhos Relacionados}

Na literatura há vários trabalhos que buscam fazer uso de sistemas de controle para tornar ambientes mais inteligentes. Esta seção descreve de forma breve alguns desses trabalhos.

O trabalho apresentado por [Silva et al. 2016] busca desenvolver um sistema inteligente para automatizar o controle e monitoramento térmico de laboratórios de análises químicas a partir de uma rede de sensores e aplicando algoritmos de Aprendizado de Máquina. Nesse sistema, um hardware construído a partir de microcontroladores ATMEGA coleta dados de temperatura do local e os envia a um banco de dados remoto.

O GREat Data Center (GDC), proposto por [Braga et al. 2017], é um sistema de monitoramento de ambientes de centros de dados. Dados obtidos por sensores são transmitidos através do protocolo ZigBee para uma placa BeagleBone, que exerce a função de gateway e armazena os dados localmente. Em seguida, os dados são retransmitidos para um servidor em nuvem, que realiza o processamento e armazenamento dos dados. Após o processamento dos dados, as informações geradas são fornecidas aos usuários através de um aplicativo para dispositivos móveis na plataforma Android.

Visando tanto o controle quanto o monitoramento, [Sabel et al. 2017] apresentam um protótipo capaz de controlar equipamentos eletrônicos pela rede. O principal componente de hardware é o módulo ESP8266EX, um chip de baixo custo com conexão Wi-Fi. Conectado a um sistema gerenciador via WebSocket, o protótipo atua sobre o ambiente de forma pré-definida de acordo com os valores aferidos pelos sensores. $\mathrm{O}$ sistema de hardware também é capaz de aferir o consumo energético de forma não invasiva, porém assumindo valores para a tensão da rede elétrica. O trabalho realizado por [Del Rio et al. 2018] também busca realizar a aplicação de um sistema baseado em Internet das Coisas (IoT) para melhorar o consumo de energia elétrica em instituições de ensino por meio da adoção o conceito de Computação em Névoa (Fog Computing) para diminuir a latência e o consumo de banda de rede.

As propostas anteriormente mencionadas apresentam conceitos interessantes relacionados com o contexto abordado neste trabalho. [Del Rio et al. 2018] apresentaram um estudo sobre o assunto, porém enfatizaram apenas a questão do consumo energético e o uso de Computação em Névoa, sem preocuparem-se com quaisquer outras problemáticas que envolvem uma implementação real do sistema. Já [Sabel et al. 2017] apresentam um protótipo mais robusto para atuação sobre os equipamentos elétricos, contudo, o equipamento é completamente dependente do sistema gerenciador. Portanto, em situações de perda de conexão à rede, o sistema ficaria inutilizável e, mesmo que fosse reestabelecida a conexão, os dados aferidos pelos sensores seriam perdidos.

A proposta de [Silva et al. 2016] em termos de uma rede de sensores conectadas a um módulo central forneceu uma detalhada descrição do processo de agrupamento e de classificação dos dados aferidos. No entanto, os autores não esclarecem qual é o papel desse elemento central (isto é, se ele somente recebe os dados dos sensores) e onde esses processos de Inteligência Artificial ocorreriam, não mencionando também em que momento se dá o controle dos ativos (aparelhos de ar condicionado e umidificadores). No caso do GDC [Braga et al. 2017], a escolha por usar módulos ZigBee torna os dispositivos locais independentes de conexão com a Internet na comunicação com o gateway, o que constitui um ponto interessante do sistema. Contudo, o BeagleBone como um gateway aparenta ser subutilizado, tendo em vista que ele não atua em nenhum momento sobre os 
equipamentos de climatização do centro de dados.

Em geral, os sistemas propostos visam apresentar uma economia real de energia elétrica, propondo uma solução que minimize a necessidade de intervenção manual e que funcione aplicada a um único caso específico. Todavia, algumas questões não são respondidas de forma unânime por esses trabalhos:

Q1: O que ocorreria se um recurso do ambiente monitorado fosse substituído?

Q2: Haveria necessidade de se ter diferentes versões de software para diferentes ambientes monitorados?

Q3: Quantas diferentes versões de hardware precisariam ser desenvolvidas para atender a todas as variações de ambientes?

Q4: Como seria feito o gerenciamento de cada unidade de hardware?

Q5: Caso a unidade de hardware esteja desconectada da plataforma central, o sistema continuaria funcionando?

Essas questões foram consideradas no desenvolvimento do Smart Place e serão discutidas na Seção 4, inclusive comparando com as propostas anteriormente relacionadas.

\section{O Smart Place}

O objetivo do Smart Place é controlar aparelhos de ar condicionado deixando-os ligados apenas quando há pessoas no ambiente monitorado ou quando há previsão de uso deste em até 15 minutos após o ambiente ter sido desocupado, uma vez que ligar e desligar o equipamento com frequência pode resultar em um maior gasto energético e contribuir para a redução de sua vida útil. Caso contrário, o aparelho deve permanecer desligado.

O sistema opera, essencialmente, a partir da integração entre um dispositivo de hardware, responsável por coletar dados referentes ao ambiente e realizar intervenções nos aparelhos de ar condicionado, e uma plataforma Web, encarregada de persistir os dados adquiridos, disponibilizá-los aos usuários do sistema e detectar eventuais anomalias no comportamento do dispositivo de hardware. A Figura 1 ilustra o dispositivo de hardware associado à visualização das informações disponibilizadas pela plataforma Web. Esses elementos são descritos nas Seções 3.1 e 3.2.

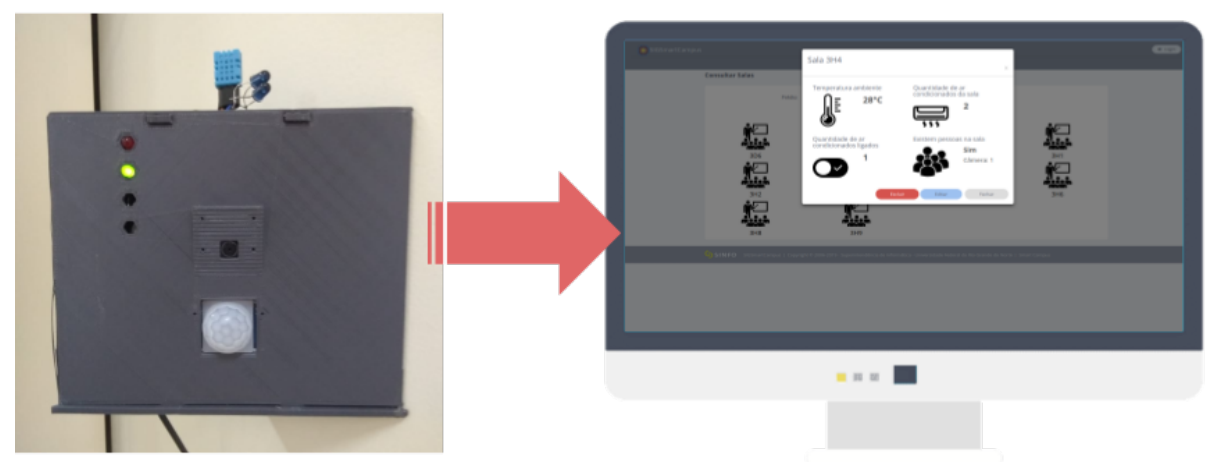

Figura 1. Dispositivo de hardware instalado em uma sala e tela de visualização da sala na plataforma Web do Smart Place.

\subsection{Dispositivo de Hardware}

O dispositivo de hardware é o elemento responsável por efetuar o controle e o monitoramento dos aparelhos de ar condicionado nos ambientes em que estão implantados. Como 
ilustra a Figura 2, tal dispositivo, desenvolvido no contexto desse trabalho, é composto por um microcomputador Raspberry Pi conectado tanto a sensores capazes de aferir dados do ambiente como movimento (1), temperatura e umidade (2), quanto a uma câmera de vídeo (3), utilizada na captura de imagens do local. Todos os dados adquiridos por tais componentes são gerenciados por esse dispositivo, que é também responsável por atuar sobre os aparelhos de ar condicionado (4), enviando sinais infravermelhos para controlar a sua operação (5). Para auxiliar no processo de decisão, os dispositivos são configurados para acessar sistemas auxiliares para consultar horários para os quais há atividades agendadas para o ambiente em questão, a exemplo de uma API provida pela Superintendência de Informática (SINFO) da UFRN ${ }^{2}$ através da qual é possível consultar os horários de aulas semanais das salas de aula da UFRN (6). As medições aferidas pelos sensores e câmeras e as ações de atuação sobre os aparelhos de ar condicionado são submetidas à plataforma Web do Smart Place (7) para que sejam persistidas e possam ser, inclusive, utilizadas por outros serviços para fins de visualização e análise.

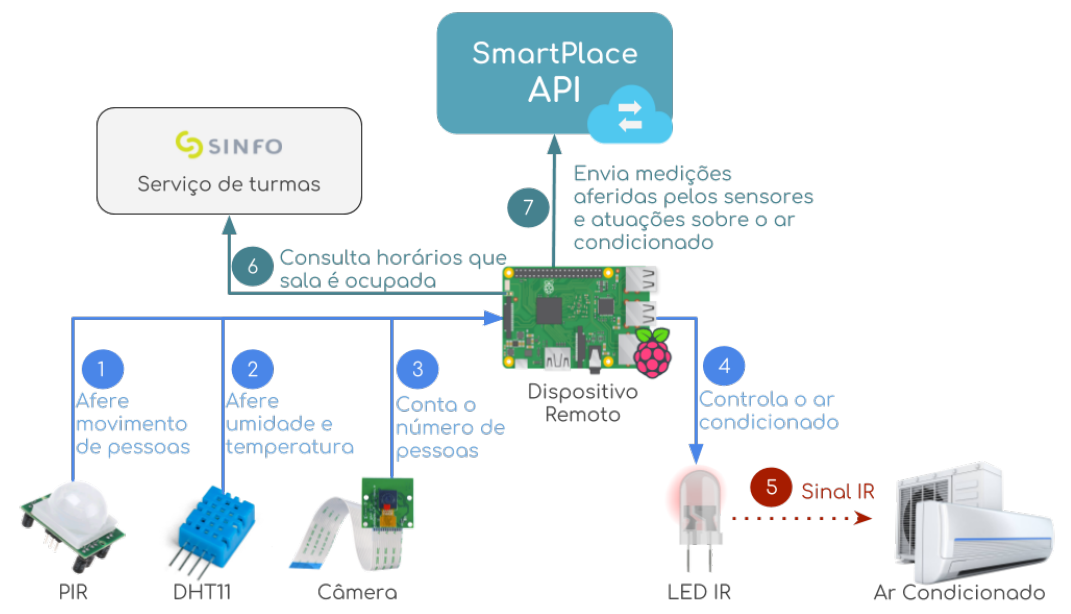

Figura 2. Visão do Smart Place na perspectiva do dispositivo remoto.

A escolha do uso da câmera de vídeo no processo de identificação de presença de pessoas em um ambiente deu-se devido a algumas limitações apresentadas pelo sensor de movimento, tais como a detecção da presença de pessoas apenas quando elas estivessem em movimento e a uma distância mínima em relação ao sensor, o que pode acabar interferindo no funcionamento do sistema. A câmera, por sua vez, fornece imagens que são usadas por um algoritmo de rede neural treinado, implantado no Raspberry Pi, que consegue detectar e contar as pessoas na sala. Entretanto, ela não substitui totalmente o sensor de movimento, pois ela também possui um rigor maior quanto a posição das pessoas e iluminação do ambiente, além de ter um tempo de resposta mais demorado e um maior consumo de recursos computacionais. Com isso, a integração desses dois elementos é uma estratégia que permite combinar a precisão da câmera com a rapidez do sensor de movimento na detecção de pessoas. É importante destacar que, para garantir a segurança e a privacidade relacionada à imagem de indivíduos, as imagens capturadas pela câmera não são persistidas de forma permanente, servindo única e exclusivamente como entrada para o algoritmo que realiza a contagem do número de pessoas presentes no ambiente

\footnotetext{
${ }^{2}$ https://api.ufrn.br/
} 
monitorado. Junto ao dispositivo de hardware há um aviso informando aos utilizadores do ambiente em questão que ele está sendo monitorado.

Além dos elementos citados, os horários de reserva dos ambientes também são considerados para a tomada de decisão. No caso de locais utilizados para aulas, que é a principal situação encontrada, o sistema executado nos dispositivos é configurado para consumir a API da SINFO para consulta de horários de aulas do local monitorado. Esses dados são armazenados localmente no Raspberry Pi para utilização offline até o fim do período letivo em questão, quando serão atualizados. Por meio desse recurso, é possível que, caso o sensor detecte movimentos dentro do horário de aula, seja dispensada a consulta de imagens da câmera para decidir quanto ao acionamento do aparelho de ar condicionado.

Para ligar ou desligar o aparelho de ar condicionado, o sistema local do dispositivo Raspberry Pi é programado para executar um conjunto de ações com base na verificação periódica (mais especificamente a cada dois segundos) do sensor de movimento. Caso seja detectado movimento, é feita uma consulta ao serviço de turmas se haverá aula no horário em questão; se sim, o aparelho de ar condicionado é ligado. Por outro lado, caso seja detectado movimento e não haja aula programada para o horário em questão, a câmera será consultada e suas imagens são processadas para contagem de pessoas no local; se a contagem de pessoas for superior a zero, o aparelho de ar condicionado é acionado. Já para decidir quanto ao desligamento do equipamento, além da verificação periódica do sensor de movimento, a câmera de vídeo captura e processa imagens a cada três segundos para a contagem do número de pessoas presentes no ambiente monitorado. Passados 15 minutos sem detecção de movimentos e o número de pessoas permanecer em zero no mesmo período, o aparelho é desligado. Caso ainda não tenha sido desligado, passados 40 minutos com a contagem de pessoas em zero, o aparelho é desligado. Com relação à regulação de temperatura da sala, a cada nova medição do sensor de temperatura (intervalo de cinco minutos) verifica-se se o valor aferido está dentro da margem de $\pm 1^{\circ} \mathrm{C}$ do valor inicialmente configurado (normalmente $23^{\circ} \mathrm{C}$ ). Caso esteja fora, o sistema emite um comando ao aparelho de ar condicionado para ajustar a temperatura.

Um aspecto essencial no desenvolvimento do Smart Place é a pluralidade das características dos ambientes de instalação do dispositivo de hardware, que faz com que diferentes necessidades sejam exigidas do sistema. Particularidades como a quantidade e os modelos dos aparelhos de ar condicionado presentes nas salas e a necessidade do uso da câmera são exemplos de requisitos dos ambientes que foram mapeados em um arquivo de configuração que é específico para cada sala e possibilita uma implementação única e genérica do controle de cada caso. Com isso, cada dispositivo instalado executa o mesmo software, tendo em vista que cada unidade possui uma especificação que é carregada no momento em que o sistema operacional do dispositivo Raspberry Pi é iniciado.

A política adotada para ambientes com maior dimensão e múltiplos aparelhos de ar condicionado é semelhante a apresentada para salas de aulas. Devido ao tamanho e a necessidade de controlar múltiplos equipamentos, é necessário utilizar múltiplos dispositivos de hardware, porém, ainda assim o sistema continua tendo a visão de um único dispositivo. Isso se dá pela distribuição das unidades controladoras em agentes com diferentes papeis, cada um sendo posicionado no ambiente para atender às necessidades. Nessa configuração, um agente assume o papel de mestre e os demais o de escravos.

Tendo em vista que replicar o mesmo dispositivo para a mesma sala implicaria em 
um custo mais elevado, os agentes escravos são mais limitados no sentido de possuírem um microcontrolador simples conectado aos sensores de movimento, umidade e temperatura. A comunicação sem fio entre os agentes, feita por meio do protocolo MQTT ${ }^{3}$, é centralizada no agente mestre, que possui um broker Mosquitto ${ }^{4}$ instalado. $\mathrm{O}$ agente escravo reporta ao agente mestre sempre que há uma nova presença em seu espaço de atuação, informando quando se passarem 15 minutos sem a detecção de presença e, regularmente, enviando dados coletados pelos outros sensores (temperatura e umidade). $\mathrm{O}$ agente mestre atua na tomada de decisões de ligar, desligar ou atualizar a temperatura do aparelho de ar condicionado em cada um dos espaços da sala. A Figura 3 apresenta como o dispositivo distribuído em agentes mestre e escravos é posicionado em uma sala para controlar os aparelhos de ar condicionado.

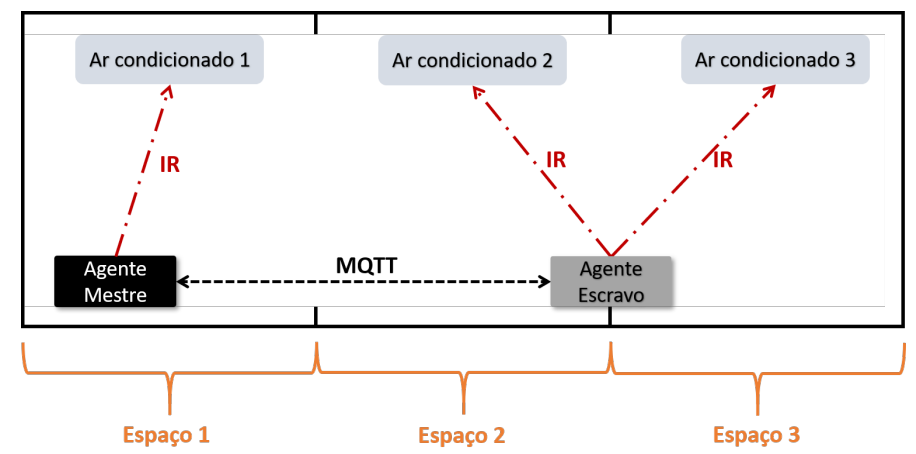

Figura 3. Mapeamento de instalação de dispositivo distribuído em um ambiente com múltiplos aparelhos de ar condicionado.

Como a sala possui múltiplos aparelhos de ar condicionado, é preciso diferenciar a lógica de acionamento adicionando uma relação de dependência entre os aparelhos. Se uma pessoa for detectada em um dos espaços físicos da sala com aparelho desligado e a margem de temperatura for aceitável (com base na temperatura ideal indicada no arquivo de configuração), é verificado se algum dos demais aparelhos está ligado. Em caso afirmativo, o aparelho do espaço ocupado pela pessoa não será ligado enquanto a margem de temperatura for aceitável. Caso nenhum dos outros aparelhos esteja ligado, o aparelho do espaço ocupado pelo indivíduo será acionado, independentemente da temperatura.

Para o evento de desligar o aparelho de ar condicionado, é modificada uma das condições padrão do dispositivo. Passados 15 minutos sem a detecção de pessoas em um dos espaços com aparelho ligado e um aparelho de ar condicionado estiver desligado em um outro espaço próximo, o aparelho do espaço com ausência de pessoas não será desligado. Essa decisão é tomada porque mais de um espaço depende desse mesmo aparelho ligado e ele só será desligado quando não houver mais espaços dependentes.

\subsection{Plataforma Web}

A plataforma Web do Smart Place corresponde a toda infraestrutura necessária para prover as funcionalidades de visualização e persistência dos dados aferidos pelo dispositivo de hardware, além de possibilitar o gerenciamento dos ambientes monitorados e a emissão de alertas para informar acerca de comportamentos inesperados. Com o uso de gráficos

\footnotetext{
${ }^{3}$ http://mqtt.org/

${ }^{4}$ https://mosquitto.org/
} 
e análises, as informações são condensadas para melhor visualização e entendimento dos dados. A plataforma Web foi construída usando a plataforma FIWARE ${ }^{5}$ como infraestrutura subjacente. Desenvolvida na Comunidade Europeia, a FIWARE foi escolhida pelo fato de ser uma plataforma genérica e de código aberto que provê diversos componentes extensíveis, reutilizáveis e interoperáveis que podem facilitar o desenvolvimento de sistemas em diferentes domínios de aplicação.

A Figura 4 ilustra a arquitetura da plataforma Web do Smart Place, composta de: (i) um Módulo Web, associado à interface gráfica com o usuário; (ii) um Módulo Central, que inclui módulos e serviços associados a autorização, notificação, controle, persistência, medições e atuações; (iii) um Módulo de Persistência para armazenamento em banco de dados; (iv) uma instância do Orion Context Broker, um componente da plataforma FIWARE que gerencia informações de contexto e permite a realização de subscrições para notificar interessados acerca de mudanças nas informações de contexto, e; (v) um Módulo de Alertas, que faz uso do Orion Context Broker para receber informações de contexto e informar ao serviço de alertas caso identifique problemas nas informações (tais como a ausência de informações sobre um dispositivo instalado na sala), possibilitando correções mais ágeis por parte dos responsáveis pela manutenção dos aparelhos.

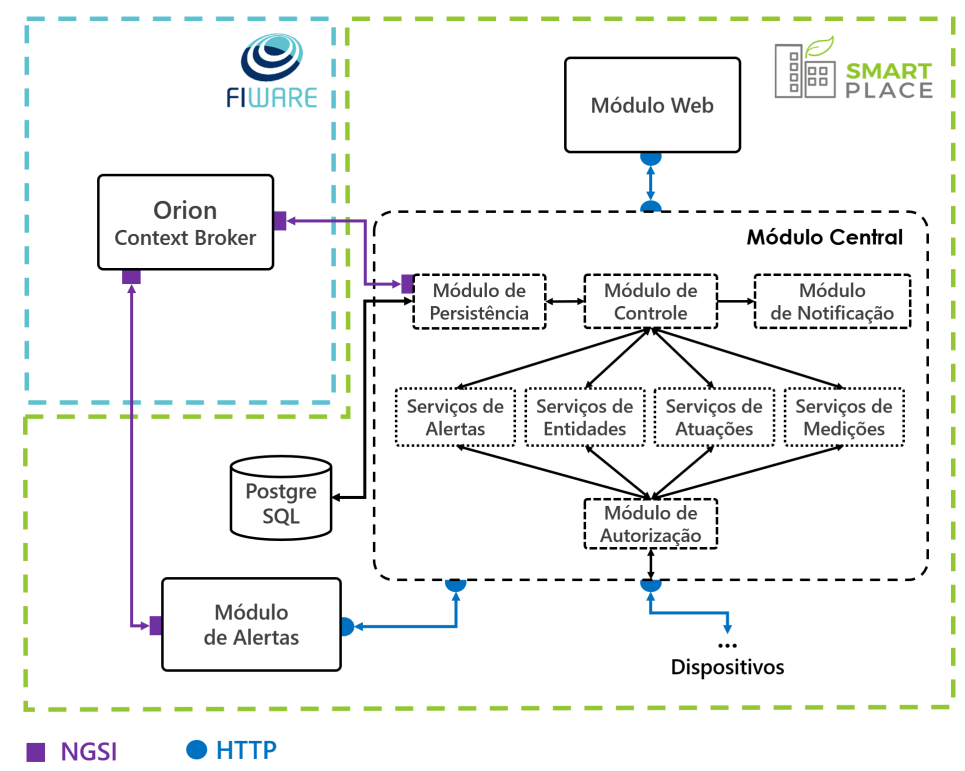

Figura 4. Arquitetura da plataforma Web do Smart Place.

No Smart Place, cada uma das salas, prédios, sensores e aparelhos de ar condicionado gerenciados estão associados a uma entidade de contexto registrada no Orion Context Broker, que mantém o registro de todas essas entidades e seus respectivos atributos de contexto, tais como potência e estado dos equipamentos, os valores aferidos pelos sensores e o instante de tempo em que foram atualizados, a que salas essas entidades estão associadas, etc. Com essas informações disponíveis no Orion Context Broker, outras aplicações podem consumi-las de forma mais eficiente, por exemplo, recebendo informações do sensor de temperatura somente quando houver variações no valor mensurado. Esse é

${ }^{5}$ https://www.fiware.org/ 
o caso do Módulo de Alertas, que é uma aplicação interessada no contexto das entidades associadas às salas, sensores e aparelhos de ar condicionado.

Através da interface gráfica fornecida pelo Módulo Web, é possível realizar operações sobre as entidades de prédios, salas, sensores e aparelhos de ar condicionado (criar, editar, excluir e visualizar), bem como visualizar as medições realizadas pelos dispositivos instalados nas salas e as intervenções sobre os equipamentos. As Figuras 5 e 6 mostram, respectivamente, um gráfico do histórico de intervenção sobre o aparelho de ar condicionado e o da contagem de pessoas presentes na sala, consultados por meio da interface disponibilizada e correspondentes aos dados enviados pelos dispositivos em um dia de atuação em uma das salas monitoradas.

\section{Histórico de Ar condicionado}

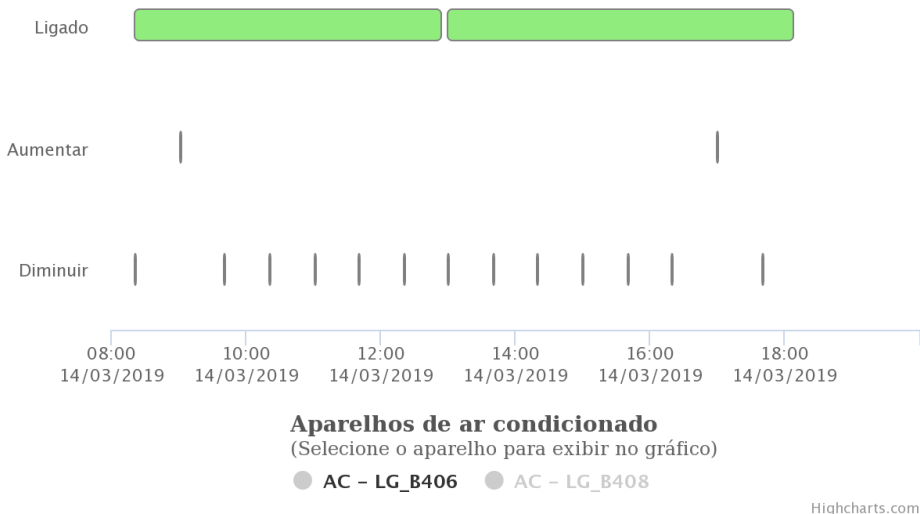

Figura 5. Gráfico de histórico de ar condicionado

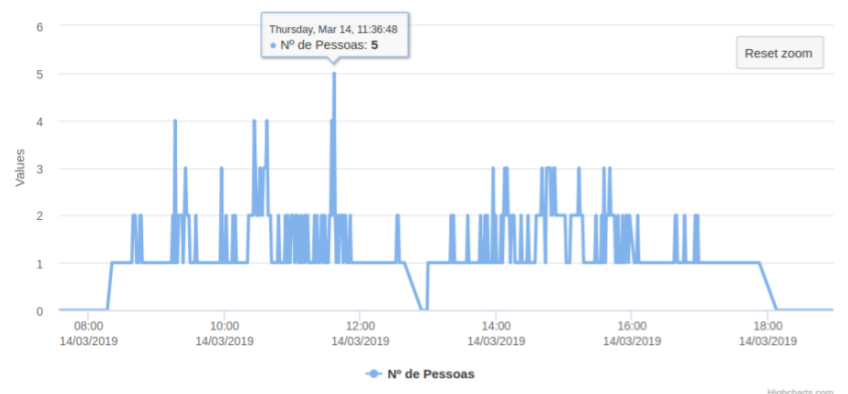

Figura 6. Visualização da número de pessoas detectadas durante um dia em uma das salas monitoradas.

$\mathrm{Na}$ Figura 5, tem-se no eixo horizontal o intervalo de datas (inicial e final definidos pelo usuário) que tenha alguma informação sobre os aparelhos de uma determinada sala. No eixo vertical estão representados os comandos que podem ser realizados pelo sistema em um aparelho de ar condicionado (ligar, desligar, aumentar temperatura, diminuir temperatura). Os comandos de ligar e desligar foram condensados em "ligado" para determinar o horário de funcionamento de cada equipamento. Na Figura 6 é apresentado graficamente o número de pessoas detectadas. Em comparação com o gráfico que mostra as intervenções sobre o aparelho de ar condicionado, é possível relacionar o período em que haviam pessoas na sala e o aparelho permaneceu acionado, sendo desligado quando as detecções apontaram que o ambiente monitorado estava vazio. 


\section{Resultados e Discussão}

O Smart Place encontra-se em produção no Campus Central da UFRN desde janeiro de 2019, tendo sido implantado um total de onze dispositivos em salas com diferentes finalidades, dentre as quais um laboratório de pesquisa, nove salas de aulas de diferentes dimensões e um escritório administrativo.

O procedimento adotado para controle dos aparelhos de ar condicionado antes da instalação dos dispositivos, o qual ainda é realizado nos lugares onde eles ainda não foram instalados, consiste na ida de funcionários em cada sala para ligar manualmente os aparelhos antes da primeira aula do dia (às 07h00) e desligá-los ao final do último horário de aula do dia (às 22h30). É importante destacar que algum aparelho pode não ser desligado ao final do dia, ficando toda a noite ligado até o dia seguinte. Além disso, a quantidade de tempo em que os aparelhos ficam ligados geralmente não condiz com a quantidade de tempo de uso efetivo dos ambientes, fazendo com que seja frequente detectar salas com aparelhos de ar condicionado ligados sem qualquer utilização, provocando um grande desperdício de energia.

Nas etapas de prototipação e implantação efetiva do Smart Place, foi possível perceber que o tempo em que os aparelhos ficam ligados geralmente não condiz com a quantidade de horas de aulas previstas para as salas, informação obtida por meio de consulta ao sistema da SINFO. O gráfico da Figura 7 apresenta um comparativo acerca do número de horas que os aparelhos de ar condicionado de uma das salas monitoradas ficam ligados nos dias úteis de uma semana, sem utilização no sábado e no domingo. Nesse gráfico é possível visualizar a quantidade de horas que o equipamento fica ligado sem a intervenção do Smart Place (ou seja, com acionamento manual), com a implantação do sistema e o número de horas previstas para utilização da sala. Com a intervenção manual, o equipamento ficou ligado por um total de 65 horas durante a semana, uma média de 13 horas por dia, enquanto que o controle automático do Smart Place permitiu reduzir o tempo de utilização para 43 horas na semana observada, com uma média diária de 8,6 horas. Isso representa uma redução de aproximadamente $33,84 \%$ no tempo total em que o aparelho de ar condicionado ficou acionado em comparação com o procedimento manual.

O benefício do uso do Smart Place era esperado em comparação à metodologia de controle manual dos aparelhos, porém se observa também uma melhoria em relação à quantidade de horas previstas para utilização da sala. Como mostrado na Figura 7, o número de horas em que o aparelho de ar condicionado permaneceu ligado ficou abaixo do número de horas previstas. Ao longo da semana observada, o Smart Place proporcionou uma redução de 6,1 horas em comparação com o total de horas em que teoricamente o aparelho ficaria acionado, representando uma economia de aproximadamente $12,4 \%$. Isso mostra a importância de um sistema de gerenciamento de ambientes como o Smart Place, capaz de detectar a presença de pessoas na sala, pois é comum as aulas começarem com atraso, terminarem antes do previsto ou até mesmo não ocorrerem.

Em relação aos trabalhos relacionados, o Smart Place é capaz de endereçar as diversas questões levantadas na Seção 2. A mudança de configuração do ambiente não acarreta diferente versão da tomada de decisão, pois cada dispositivo é carregado com uma descrição dos recursos do ambiente e o caso da substituição de um aparelho de ar por um de diferente modelo é facilmente resolvida com uma alteração no arquivo de descrição utilizado pelo dispositivo instalado na sala (Q1). A mesma estratégia garante que 


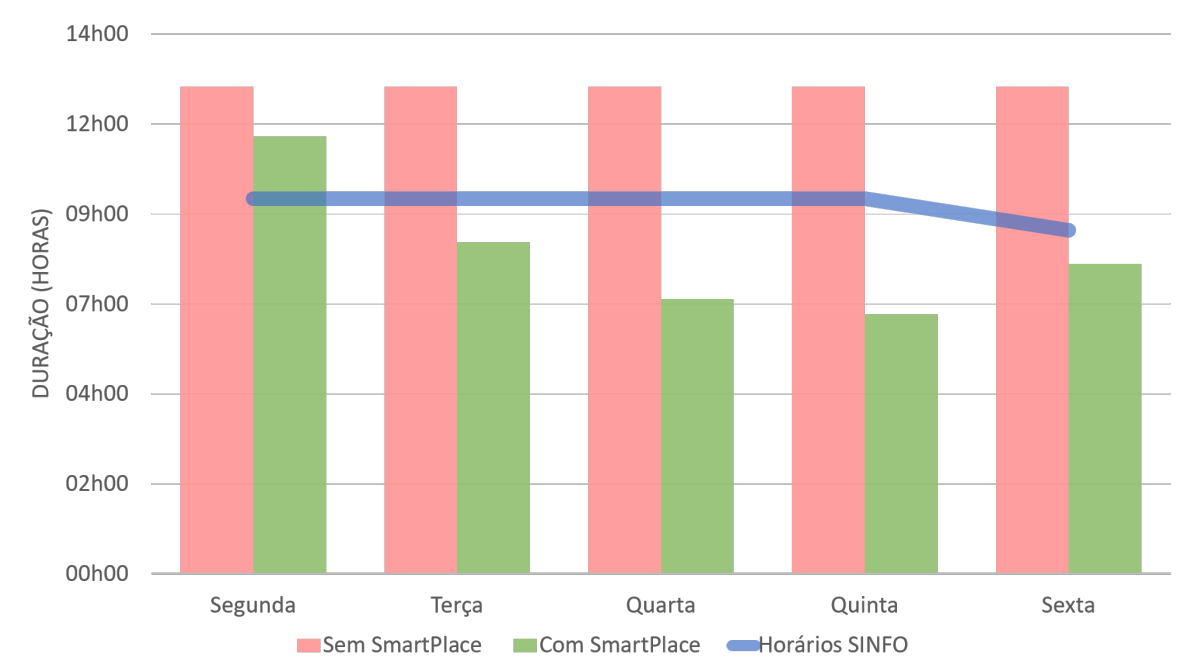

Figura 7. Comparativo do número de horas de funcionamento dos aparelhos de ar condicionado considerando intervenção manual, intervenção automática pelo Smart Place e o número de horas previstas para utilização da sala.

haja uma única versão do software para diferentes tipos de ambientes (Q2) e, ainda que haja variações em termos de hardware, é possível endereçar essa questão distribuindo o monitoramento na forma de agentes mestre-escravo (Q3). Quanto ao problema de gerenciamento dos dispositivos remotos, este é solucionado com o Módulo de Alertas, no qual as inconformidades são automaticamente identificadas e os administradores são notificados por $e$-mail para procederem com correções (Q4). Por fim, o Smart Place permite que a tomada de decisão pelos dispositivos seja independente de conectividade, ainda que o acesso a sistemas externos (a exemplo do serviço de consulta de horários de aula) aumente o grau de confiança das decisões de atuação sobre os aparelhos de ar condicionado (Q5).

\section{Conclusão}

O presente trabalho apresentou o Smart Place, um sistema de gerenciamento de ambientes voltado para o controle automatizado de aparelhos de ar condicionado no intuito de contribuir para a diminuição do desperdício de energia elétrica. Nesse sistema, um dispositivo de hardware com sensores e câmera de vídeo coleta dados referentes a temperatura, umidade e presença de pessoas nos ambientes monitorados, de modo que tais dados são utilizados para realizar intervenções nos aparelhos de ar condicionado, ligando e desligando-os quando necessário. Além disso, uma plataforma Web encarrega-se da persistência e disponibilização dos dados adquiridos, além de possibilitar a detecção de eventuais anomalias. O Smart Place está atualmente em operação monitorando um total de onze salas e laboratórios do Campus Central da UFRN, em Natal-RN.

Este artigo também apresentou alguns resultados decorrentes da observação da quantidade de horas de funcionamento dos aparelhos de ar condicionado em uma das salas monitoradas, contrastando intervenções manuais (como é tradicionalmente feito) e intervenções automáticas pelo Smart Place. Com base nesses resultados, é possível concluir que o sistema consegue atingir o objetivo proposto para o seu desenvolvimento, que é contribuir com o desperdício de energia elétrica por evitar que os equipamentos permaneçam ligados em situações em que não estão sendo utilizados. Mais ainda, o trabalho fornece contribuições no sentido que é capaz de endereçar algumas limitações identifica- 
das em soluções existentes.

Atualmente, a implantação do Smart Place continua em expansão para outros prédios e salas de aula da Universidade. Em trabalhos futuros, será realizada uma avaliação que explore aspectos de desempenho, escalabilidade e resiliência do sistema. Por meio dessa maior escala de implementação, será possível verificar não apenas como o sistema se comportaria sob um grande número de requisições e dados gerenciados, mas também aferir o real impacto da adoção do Smart Place para alcançar seu principal objetivo, que é contribuir com a eficiência energética. Por fim, planeja-se integrar o Smart Place com outra solução em desenvolvimento na Universidade para o monitoramento efetivo do consumo de energia elétrica, permitindo assim aferir o quanto de economia foi possível proporcionar e qual o impacto em termos de redução de custos.

\section{Referências}

ABESCO (2017). Desperdício de energia atinge $\mathrm{R} \$ 61,7$ bi em três anos. Disponível em: <http://www.abesco.com.br/pt/novidade/ desperdicio-de-energia-atinge-r-617-bi-em-tres-anos $>$.

Berriel, R. C. d. O. (2017). Eficiência energética no Brasil e diagnóstico energético para consumidores do Grupo Tarifário B Convencional - Estudo de caso SUIPA. Projeto de Graduação, Universidade Federal do Rio de Janeiro, Brasil.

Braga, A. R., Maciel, F. A. O., Almeida, R. L. A., Aguilar, P. A. C., Gomes, D. G., Andrade, R. M. C. (2017). Gerenciamento térmico e elétrico de um centro de dados utilizando sensoriamento IoT. In Anais do XXXVII Congresso da Sociedade Brasileira de Computação, páginas 998-1007, Porto Alegre, Brasil. SBC.

Del Rio, L. S., Silva, J. O. B., Azevedo, M. d. S., Pereira, É. P., Fischer, I. A., Medina, R. D. (2018). Proposta de ambientes inteligentes IoT sob a ótica da eficiência energética. In Anais do Encontro Anual de Tecnologia da Informação, páginas 86-93.

Sabel, G., Wisintainer, M., Péricas, F. A., Marcelo Mattos, M. (2017). Sistema de gestão de energia elétrica em salas de aula de uma universidade baseado em IoT. Revista de Sistemas e Computação, 7(2):385-405.

Silva, A. R., Xavier Júnior, J. C., Silva, I. (2016). Rede de sensores para controle inteligente de ambientes. In Anais do XXXVI Congresso da Sociedade Brasileira de Computação, páginas 1096-1105, Porto Alegre, Brasil. SBC. 